Aim of the study: To assess influencing factors and main health-related quality of life (HRQoL) issues in patients with cancers of the oesophago-gastric region using the European Organization for Research and Treatment of Cancer (EORTC) quality of life questionnaire Core 30 (QLQ-C30) and its oesophago-gastric module (QLQ-OG25).

Material and methods: Patients were qualified for this study based on the histological confirmation of oesophageal, oesophago-gastric or gastric cancers. Each patient filled out the Polish version of the EORTC QLQ-C30, the QLQ-OG25 module and a personal questionnaire. Patients were divided into groups based on gender, age, treatment intention, tumour localization, working status and level of education.

Results: Our study included 112 patients - 39 women (35\%) and 73 men (mean age $\pm S D ; 60.2 \pm 10.9$ ). Thirty-five patients (31.3\%) completed the questionnaires twice. Eighty-four (75\%) patients had gastric cancer (GC), twenty-six (23.2\%) oesophageal cancer (OC) and two (1.8\%) cancer of the oesophago-gastric junction (OGJC). Eighty (71.4\%) patients underwent surgical treatment prior to either chemo-, radio- or chemoradiotherapy. The Global Health Status scale of the QLQC30 inversely correlated with all the other QLQ-C30 and QLQ-OG25 symptom scales $(r=-0.26$ to $-0.61 ; p<0.05)$. Conclusions: The main HRQoL problems of Polish OC, OGJC and GC patients are fatigue, insomnia, anxiety, and appetite and weight loss. Older age, receiving palliative treatment, having gastric cancer, being on retirement and having lower education are factors associated with higher symptom scores (worse symptoms) and thus poorer HRQoL.

Key words: cancer, oncology, quality of life, QLQ-OG25, QLQ-C30, oesophagogastric.

\section{Main influencing factors and health- related quality of life issues in patients with oesophago-gastric cancer - as measured by EORTC tools}

\author{
Krzysztof A. Tomaszewski ${ }^{1}$, Mirosława Püsküllüoğlu', Katarzyna Biesiada ${ }^{3}$, \\ Justyna Bochenek ${ }^{2}$, Sebastian Ochenduszko², Iwona M. Tomaszewska ${ }^{4}$, \\ Krzysztof Krzemieniecki ${ }^{2}$
}

1Department of Anatomy, Jagiellonian University Medical College, Krakow, Poland 2Department of Clinical Oncology, University Hospital, Krakow, Poland ${ }^{3}$ Department of Internal Medicine, Bonifratres Hospital, Krakow, Poland ${ }^{4}$ Department of Prosthetic Dentistry, Institute of Dentistry, Jagiellonian University Medical College, Krakow, Poland

\section{Introduction}

Over the last three decades the epidemiology of oesophageal and gastric cancers has demonstrated significant changes [1]. In Poland cancers of the oesophagus (OC) and the oesophago-gastric junction (OGJC) are rare-below $2 \%$ of all malignant neoplasms [2]. In men oesophageal cancer is the 10th cause (2.3\%) of cancer-related deaths. On the other hand, gastric cancers are still fairly common in Poland and constitute $5.7 \%$ and $3.4 \%$ of all malignant neoplasms in Polish men and women respectively. Gastric cancer remains the $3^{\text {rd }}$ ( $7.4 \%$ in $\mathrm{men}$ ) and $5^{\text {th }}$ (5.5\% in women) cause of cancer-related deaths [3]. Thus GC in Poland still represents a huge social and oncological problem.

Worldwide the incidence of $O C$ and gastric cardia cancers is increasing, while the incidence of non-cardia adenocarcinomas of the stomach is decreasing [1]. Health-related quality of life (HRQoL) measurement is an important component of the assessment of cancer patients, including patients with OC, OGJC and gastric malignancies. Having in mind the number of people with oesophago-gastric cancers, we should understand that HRQoL problems in this group of patients currently represent a major challenge, firstly, because of the nature of the disease, and secondly, because of the number of people suffering from oesophago-gastric region cancers. Not only is HRQoL an important outcome measurement in everyday oncological practice, but also it is more and more commonly taken into account as one of the endpoints in clinical trials, providing a lot of information about the state of our patients [4, 5]. For some patients, especially with metastatic disease, it is the most important thing, even more important than prolonging life. That is why it is imperative to get to know the main HRQoL issues which oesophago-gastric cancer patients have to face every day. This knowledge will help physicians meet the needs of their cancer patients. There are studies stating that by simply measuring HRQoL also the patient-physician communication may be improved [6].

Quality of life questionnaires (QLQ) for measuring HRQoL in cancer patients are created by the European Organization for Research and Treatment of Cancer (EORTC). There are questionnaires designed to measure HRQOL in OC and GC patients. The QLQ-C3O is the core questionnaire and there are additional modules that are meant to be used with it [7]. Those are the QLQSTO22, specific for gastric cancers [8]; the QLQ-OES18, specific for oesophageal cancers [9]; and the QLQ-OG25 (created by combining the corresponding ques- 
tions from the previous two), specific for oesophago-gastric cancers [10]. By using the QLQ-OG25 instead of two separate questionnaires, one can avoid the existing overlapping in HRQoL issues between oesophageal and gastric cancer patients. This has decreased the number of questions the patient has to answer during the interview. All of the questionnaires have been translated and validated into many languages and their Polish versions are available from the EORTC $[10,11]$. Our group has translated and validated the Polish version of the QLQ-OG25 module [12].

The aim of this study was to assess the influencing factors and main health-related quality of life issues in patients with cancers of the oesophago-gastric region using the EORTC QLQ-C30 and its QLQ-OG25 module.

\section{Material and methods}

\section{Patients}

In this study patients were recruited prospectively between January 2011 and May 2012. The recruitment process took place at the Department of Clinical Oncology at the University Hospital in Krakow. Patients were eligible if they had a histologically confirmed diagnosis of oesophageal or gastric cancer, including neoplasms located at the oesophagogastric junction. Location of the tumour was reconfirmed on the basis of computed tomography (CT). All histological types of OC, GC and OGJC were included in the study.

Patients included in the study were classified into groups based on gender (female vs. male), age (below vs. above or equal to 60 years of age), treatment intention (curative vs. palliative), tumour localization (oesophagus vs. stomach) and working status (employed vs. retired/pensioner).

Patients were interviewed during their visits at the outpatient clinic or during their stay at the ward. The second interview took place after a minimum of two weeks counting from the first interview. During the interview each patient completed the Polish version of the EORTC QLQ-C30, the QLQOG25 module and a personal questionnaire. Clinical history and physical examination were registered for all patients.

Exclusion criteria included lack of consent to participate in the study, inability to understand or complete the questionnaires and presence of concurrent malignancies. There were no restrictions as to gender or age. All patients gave their informed consent to participate in this study. The research protocol was approved by the Jagiellonian University Ethics Committee (registry KBET/250/B/2011).

\section{Translation and pilot testing}

The Polish version of the EORTC QLQ-C30 (version 3.0) was previously translated. The translation process of the EORTC QLQ-OG25 Polish version was authorized by the EORTC. Items deriving from the Polish versions of the QLQ-STO22 and the QLQ-OES18 were used in an unchanged state. The remaining items were translated according to the official EORTC guidelines [13].

The translated, initial version of the QLQ-OG25 was pilottested on 25 Polish patients with OC, OGJC or GC. Their comments were analyzed and minor language changes were made to the initial translation. This version was once again tested on 15 additional patients. After this phase the final transla- tion of the Polish version of the QLQ-OG25 was obtained and approved by the EORTC Quality of Life Department.

\section{Questionnaires}

The QLQ-C30 is a 30-item questionnaire comprised of a global health status, five multi-item functional scales, three multi-item symptom scales, and six symptom single items.

The QLQ-OG25 is a 25-item module comprised of six multi-item scales: dysphagia, eating restriction, reflux, odynophagia, pain and discomfort, and anxiety. In addition to the six multi-item scales the QLQ-OG25 has 10 single items. Higher scores indicate worse symptomatic problems.

All of the QLQ-C30 and the QLQ-OG25 multi-item scales and single items were scored on a 1 to 4 point Likert scale ("not at all", "a little", "quite a bit", "very much"), apart from items 29 and 30 of the QLQ-C30, which were scored on a 1 to 7 point scale. Items 29 and 30 comprise the global health status. Detailed information on how to score the EORTC questionnaires can be found in the QLQ-C30 scoring manual and its addendum [14].

\section{Statistical analysis}

Statistical analysis was conducted using Statistica 10.0 PL computer software by StatSoft Poland. Scores for multiitem functional or symptom scales and for single items were calculated by linear transformation of raw scores into a 0 100 score, with 100 representing the best global health, functional status or worst symptoms - depending on the measuring property of each multi-item or single-item scale, as described by the EORTC $[14,15]$. To analyse the data we used elements of descriptive statistics (mean, standard deviation, percentage distribution).

Group comparison was used to assess the differences in HRQoL issues between known groups. Known groups used in this study were: gender (female vs. male), age (below vs. above or equal to 60 years of age), treatment intention (curative vs. palliative), tumour localization (oesophagus vs. stomach), working status (employed vs. retired/pensioner) and level of education (elementary vs. high school vs. vocational vs. university). Differences between groups were tested with ANOVA, t-test and Mann-Whitney tests as appropriate.

Spearman correlation analysis was performed to explore the relationship between scales of the QLQ-C30 and the QLQOG25.

A subset of patients completed the questionnaires twice $(n=35)$ to evaluate whether HRQoL issues can change over a short period of time. The significance level was set at $p<0.05$

\section{Results}

\section{Patient characteristics}

A total of 112 patients were recruited into the study 39 women (35\%) and 73 men. Mean age of the group was 60.2 years \pm standard deviation (SD) of 10.9 years. Eightyfour patients had gastric cancer, twenty-six oesophageal cancer and two cancer of the oesophago-gastric junction. Patients' clinical and demographic data are presented in Table 1. Eighty (71.4\%) patients underwent surgical treatment prior to either chemo-, radio- or chemoradiotherapy. 
Table 1. Patients' demographic and clinical data

\begin{tabular}{|c|c|c|c|}
\hline & $\begin{array}{l}\text { Overall } \\
(n=112)\end{array}$ & $\begin{array}{l}\text { Curative treatment } \\
\qquad(n=52)\end{array}$ & $\begin{array}{l}\text { Palliative treatment } \\
\qquad(n=60)\end{array}$ \\
\hline \multicolumn{4}{|l|}{ Gender (\%) } \\
\hline Female & $39(35 \%)$ & 19 (36.5\%) & $20(33.3 \%)$ \\
\hline Male & $73(65 \%)$ & $33(63.5 \%)$ & $40(66.7 \%)$ \\
\hline Age (SD) & $60.2(10.9)$ & $59.8(9.5)$ & $61.2(12.5)$ \\
\hline \multicolumn{4}{|l|}{ Cancer site (\%) } \\
\hline Oesophagus & $26(23.2 \%)$ & $8(15.3 \%)$ & $18(30 \%)$ \\
\hline Stomach & $84(75 \%)$ & $42(81 \%)$ & $42(70 \%)$ \\
\hline Oesophago-gastric junction & $2(1.8 \%)$ & $2(3.7 \%)$ & 0 \\
\hline \multicolumn{4}{|l|}{ Education (\%) } \\
\hline Elementary & $20(17.9 \%)$ & $10(19.2 \%)$ & $10(16.7 \%)$ \\
\hline High school & $28(25 \%)$ & $12(23.1 \%)$ & $16(26.7 \%)$ \\
\hline Vocational & $46(41.1 \%)$ & $20(38.5 \%)$ & $26(43.3 \%)$ \\
\hline University & $18(16 \%)$ & $10(19.2 \%)$ & $8(13.3 \%)$ \\
\hline \multicolumn{4}{|l|}{ Employment (\%) } \\
\hline Full time/part time & $63(56.3 \%)$ & $40(77 \%)$ & $23(38.3 \%)$ \\
\hline Retired/Pensioner & $49(43.7 \%)$ & $12(23 \%)$ & $37(61.7 \%)$ \\
\hline
\end{tabular}

No patients rejected taking part in the study. All 112 patients answered both the QLQ-C30 and the QLQ-OG25. Thirty-five patients were selected to complete the questionnaires a second time after a minimum of 2 weeks following the first interview.

\section{Main health-related quality-of-life issues}

When taking into account the whole patient group the main HRQoL issues (basing on the QLQ-C30) were Fatigue (49/100 points), Insomnia (42.7/100 points), Appetite Loss (41.7/100 points) and Role Functioning (40.1/100 points). The scale assessing Diarrhoea (16.4/100 points) had the smallest impact on the overall HRQOL.

Based on the QLQ-OG25 the scales assessing Anxiety (58.3/100 points), Weight Loss (45.5/100 points), Body Image (43.6/100 points) and Eating Restriction (37.6/100 points) had the biggest impact on the overall HRQoL The scale assessing Trouble Talking (6.9/100 points) had the smallest impact on the overall HRQoL.

\section{Group comparison}

Comparing overall scores from the first and second interview, one can say that HRQoL in patients with OC, OGJC and GC does not change over a short period of time $(p>0.05)$.

Comparison by gender shows that scales and items distinguishing between sexes are Pain (31.8 vs. 20; $p=0.0223$ ), Diarrhoea (22 vs. 4.8; $p=0.0076$ ), Odynophagia (22.5 vs. 11.1; $p=0,0144$ ) (men having significantly higher scores) and Hair Loss (10.2 vs. 41.6; $p=0.0010$ ) (women having significantly higher scores).

Known group comparison by age is shown in Table 2 . Scales and items that distinguish $(p<0.05)$ between age

Table 2. Group comparison by age. Only statistically significant data are shown

\begin{tabular}{|c|c|c|c|c|c|c|c|}
\hline \multirow[b]{2}{*}{ Scale/Item } & \multicolumn{3}{|c|}{ Age $<60$} & \multicolumn{3}{|c|}{ Age $\geq 60$} & \multirow[b]{2}{*}{$p$-value } \\
\hline & $n$ & Mean & SD & $n$ & Mean & SD & \\
\hline Physical Functioning & 53 & 45.8 & 13.7 & 59 & 61.8 & 27.7 & 0.0002 \\
\hline Fatigue & 53 & 41.9 & 21.4 & 59 & 55.2 & 26.3 & 0.0043 \\
\hline Nausea and Vomiting & 53 & 8.3 & 12.2 & 59 & 30.0 & 31.8 & $<0.0001$ \\
\hline Pain & 53 & 20.0 & 27.1 & 59 & 35.4 & 30.6 & 0.0059 \\
\hline Appetite Loss & 53 & 28.9 & 32.4 & 59 & 52.9 & 38.6 & 0.0006 \\
\hline Eating Restriction & 53 & 26.9 & 25.3 & 59 & 47.1 & 27.5 & 0.0001 \\
\hline Pain and Discomfort & 53 & 15.0 & 21.6 & 59 & 31.4 & 31.7 & 0.0020 \\
\hline
\end{tabular}


Table 3. Group comparison by tumour location. Only statistically significant data are shown

\begin{tabular}{|c|c|c|c|c|c|c|c|}
\hline \multirow[b]{2}{*}{ Scale/Item } & \multicolumn{3}{|c|}{ Stomach } & \multicolumn{3}{|c|}{ Oesophagus } & \multirow[b]{2}{*}{$p$-value } \\
\hline & $n$ & Mean & $\overline{S D}$ & $n$ & Mean & $\overline{S D}$ & \\
\hline Global Health Status & 84 & 63.6 & 42.3 & 26 & 41.2 & 43.9 & 0.0212 \\
\hline Role Functioning & 84 & 59.0 & 37.0 & 26 & 35.3 & 31.4 & 0.0039 \\
\hline Pain & 84 & 41.0 & 30.9 & 26 & 24.7 & 28.8 & 0.0187 \\
\hline Dyspnoea & 84 & 51.3 & 42.2 & 26 & 19.6 & 27.6 & 0.0005 \\
\hline Insomnia & 84 & 61.5 & 18.5 & 26 & 37.9 & 30.6 & $<0.0001$ \\
\hline Appetite Loss & 84 & 61.5 & 30.0 & 26 & 36.6 & 37.9 & 0.0008 \\
\hline Dysphagia & 84 & 49.1 & 33.7 & 26 & 19.2 & 24.9 & $<0.0001$ \\
\hline Eating Restriction & 84 & 54.2 & 20.9 & 26 & 33.8 & 28.4 & 0.0001 \\
\hline Odynophagia & 84 & 32.0 & 25.1 & 26 & 15.7 & 25.0 & 0.0046 \\
\hline Eating with Others & 84 & 38.9 & 37.2 & 26 & 9.3 & 20.3 & 0.0002 \\
\hline Trouble Swallowing Saliva & 84 & 44.4 & 38.5 & 26 & 5.8 & 17.1 & $<0.0001$ \\
\hline Trouble with Coughing & 84 & 55.6 & 38.5 & 26 & 13.5 & 20.1 & $<0.0001$ \\
\hline Trouble Talking & 84 & 25.0 & 37.9 & 26 & 2.6 & 11.2 & 0.0037 \\
\hline Choking when Swallowing & 84 & 27.8 & 31.3 & 26 & 5.8 & 15.8 & 0.0008 \\
\hline Weight Loss & 84 & 63.9 & 33.2 & 26 & 41.2 & 36.9 & 0.0037 \\
\hline
\end{tabular}

SD - standard deviation

Table 4. Group comparison by working status. Only statistically significant data are shown

\begin{tabular}{|c|c|c|c|c|c|c|c|}
\hline \multirow[b]{2}{*}{ Scale/Item } & \multicolumn{3}{|c|}{ Working } & \multicolumn{3}{|c|}{ Retired } & \multirow[b]{2}{*}{$p$-value } \\
\hline & $n$ & Mean & SD & $n$ & Mean & $\mathrm{SD}$ & \\
\hline Physical Functioning & 63 & 42.5 & 17.7 & 49 & 31.2 & 28.1 & 0.0128 \\
\hline Fatigue & 63 & 42.6 & 23.2 & 49 & 57.6 & 24.9 & 0.0014 \\
\hline Appetite Loss & 63 & 31.5 & 34.2 & 49 & 55.6 & 38.1 & 0.0006 \\
\hline Constipation & 63 & 18.9 & 25.5 & 49 & 40.5 & 39.9 & 0.0007 \\
\hline Dysphagia & 63 & 17.0 & 23.1 & 49 & 34.9 & 32.8 & 0.0010 \\
\hline Eating Restriction & 63 & 30.3 & 25.8 & 49 & 47.0 & 28.7 & 0.0014 \\
\hline Pain and Discomfort & 63 & 17.1 & 23.4 & 49 & 32.1 & 32.4 & 0.0053 \\
\hline Dry Mouth & 63 & 25.9 & 29.9 & 49 & 44.1 & 36.4 & 0.0044 \\
\hline Trouble with Coughing & 63 & 15.7 & 28.2 & 49 & 28.6 & 29.7 & 0.0207 \\
\hline
\end{tabular}

SD - standard deviation

groups are Physical Functioning, Fatigue, Nausea and Vomiting, Pain, Appetite Loss, Eating Restriction, and Pain and Discomfort. Overall older age was associated with higher symptom scores.

Known group comparisons by treatment intention showed that patients in the curative intention group versus patients in the palliative treatment group present lower scores (less symptoms) on the Physical Functioning (25.6 vs. 42; $p=0.0147$ ), Fatigue (40 vs. 56.8; $p=0.0058)$, Insomnia (34.4 vs. 50; $p=0.0372$ ) and Appetite Loss (30 vs. 52; $p=0.0183$ ) scales. No significant differences between the curative and the palliative treatment group were noted in any of the scores of the QLQ-OG25 scales or items.

Table 3 presents mean scores according to tumour location. As for the QLQ-C30, scales that distinguished $(p<0.05)$ between the two groups were Global Health Status and Role Functioning. Single items of the QLQ-C30 that distinguished between the two groups were Pain, Dyspnoea, Insomnia and Appetite Loss. As for the QLQ-OG25, scales that distinguished between the two groups were Dysphagia, Eating Restriction, and Odynophagia. Single items of the QLQ-OG25 that distinguished between the two groups were Eating with Others, Trouble Swallowing Saliva, Trouble with Coughing, Trouble with Talking, Choking when Swallowing and Weight Loss.

When scores were calculated by working status (Table 4) both the QLQ-C30 and the QLQ-OG25 showed significantly lower scores (less symptoms) in patients who are still professionally active.

Comparing patient scores by level of education (elementary vs. high school vs. vocational vs. university) showed that 
patients with university level education cope better with pain (Pain scale of the QLQ-C30) than patients with less education $(p=0.0482)$. Scores from other scales presented no significant differences in HRQoL associated with level of education ( $p>0.05$ ) but a trend was observed suggesting that higher education is associated with lower symptom scores.

\section{Correlations between the QLQ-C30 and the QLQ-OG25}

The Global Health Status scale of the QLQ-C30 inversely correlated with all the other QLQ-C30 and QLQ-OG25 symptom scales $(r=-0.28$ to $-0.61 ; p<0.05)$. An especially strong, inverse correlation was noted between the Global Health Status and Fatigue scales $(r=-0.61 ; p<0.0001)$.

Strong correlations were also noted between the Nausea and Vomiting scale of the QLQ-C30 and the Dysphagia and Eating Restriction scales of the QLQ-OG25 ( $r=0.63$ and $r=0.65$ respectively; $p<0.0001$ ).

Three scales of the QLQ-OG25 correlated strongly with each other - Dysphagia, Eating restriction and Odynophagia $(r=0.62-0.75 ; p<0.0001)$.

\section{Discussion}

This study presents influencing factors and main HRQoL issues in oesophago-gastric cancer patients as measured by EORTC tools - the QLQ-C30 and the QLQ-OG25.

Measuring HRQOL is becoming an important outcome in clinical trials and daily clinical practice. Several studies have already used patient-reported outcome measures to assess HRQoL in patients with oesophageal, gastric and oesophago-gastric junction cancers [16-18].

Taking into account the obtained results, firstly we should remember that cancer patient HRQoL problems should not be viewed in a short-term perspective. As we can see, a two to four week period is not enough to observe significant changes in HRQoL.

Our study shows that women tend to experience less severe (or admit to experiencing less severe) symptoms than men. This is especially visible in scales such as Pain, Diarrhoea and Odynophagia. Our results stand in contradiction to those obtained by van der Schaaf et al. [19]. We lack a good explanation as to why such gender-dependent symptom changes exist. For example, laboratory studies conducted thus far on humans have showed no significant differences in pain perception among men and women [20]. On the other hand, women, compared to men, seem to focus more on appearance issues such as hair loss, which is consistent with the culturally based idea of women wanting to feel more visually attractive than men. Similar gender-related differences in EORTC questionnaire scale scores were noted in other studies [21].

Age had a substantial impact on the amount and severity of symptoms. The overall trend was that older age was associated with higher symptom scores, but not all scales achieved statistical significance. We have found this to be consistent with other HRQoL studies [19, 22].

When comparing HRQoL by tumour location, patients with stomach cancer consistently present higher symptom scores (higher severity of symptoms), even when it comes to symptoms specific for oesophageal cancer such as "Trouble Swal- lowing Saliva" or "Choking when Swallowing". This partially stands in contradiction to data presented by Onate-Oceana et al. [23], which shows that as far as the above mentioned symptoms are concerned, they are more specific for oesophageal cancer patients than for gastric cancer patients. It is impossible to argue which type of cancer would cause more severe symptoms. This relies rather on the stage of the disease, not the disease type.

When taking into account professional activity, we observed a trend that patients remaining professionally active present less severe symptoms than patients on retirement. This is not so easily explained. First of all, in the case of our study it is impossible to determine whether being professionally active prevents development of severe symptoms or whether the development of severe symptoms forces a patient to retire. Secondly, our "retired patient" group has a higher mean age and that can also contribute to increased symptom severity as described earlier. Hence the difficulties in establishing the correct chain of events that creates the base for more severe symptoms to develop in patients on retirement. Nevertheless, our findings concerning the role of professional activity among cancer patients are consistent with literature data [24].

Analysing the obtained correlations we can see that Fatigue is the factor with the strongest impact on overall health status. We think it is so because fatigue, at least in our cancer patient group, was the symptom most often reported and also associated with the highest number of additional problems. Other factors that most significantly affected the health status were Appetite loss and Eating restriction. All three of those elements - Fatigue, Appetite loss and Eating restriction - are related to the cachexia-anorexia syndrome. Studies conducted among patients with malignancies including cancers of the upper gastrointestinal tract showed that the severity of cachexia-anorexia has a huge impact on the quality of life in these patients [25, 26].

The strong correlation between the Dysphagia, Eating restriction and Odynophagia scales can be explained by the fact that patients often merged these three problems into one, even when supplied with additional information from the interviewer.

What is interesting, patients attribute most of their HRQoL issues to problems due to fatigue, anxiety, insomnia, body image, appetite and weight loss. This is partially consistent with other studies that show that especially anxiety is a major problem among cancer patients [23].

Anxiety, insomnia, appetite loss and body image can be considered more as psychological issues than purely medical ones. Symptoms such as dysphagia, reflux, odynophagia, nausea and vomiting are ranked lower and do not contribute as much to the overall HRQoL score as psychological problems. This points to the fact that Polish cancer patient care should focus even more on patient psychological problems.

To allow for a comparison of the data from this manuscript (results from a single-centre study) to the general population, certain comments, on the subject of study group characteristics, are needed. A study by Krstev et al. [27], conducted on 443 Polish patients with newly diagnosed gastric cancer, shows similar data in regard to gender distribution (35.7\% women) and mean age (mean age of 63.8) of participants. 
This points to the fact that the results obtained in this study can be related to the general Polish population. Though the incidence of GC in Poland is predicted to decrease [28] in the next 12 years, this cancer will still be more common in men then in women. The fall in the incidence of GC will be notably slower in the group of people older then 65 years [28]. Thus, this age group will remain the dominant one among Polish GC patients. The GC gender percentage distribution is not predicted to change [28].

In conclusion, the main HRQoL problems of Polish OC, OGJC and GC patients are fatigue, insomnia, anxiety, appetite and weight loss. Polish cancer patients report fewer organic HRQoL issues then their western European counterparts. Older age, receiving palliative treatment, having a cancer of the stomach, being on retirement and having lower education are factors associated with higher symptom scores (worse symptoms) and thus poorer health-related quality-of-life.

The authors declare no conflict of interest.

The authors would like to thank Jolanta Biesiada MD and Bartosz Wilk MD for supporting this study. We also thank the European Organization for Research and Treatment of Cancer Quality of Life Department for support during the translation process of the QLQ-OG25.

This study has not been funded.

\section{References}

1. Botterwecka AM, Schoutenb LJ, Volovicsc A, Doranta E, van den Brand ta PA. Trends in incidence of adenocarcinoma of the oesophagus and gastric cardia in ten European countries. Int J Epidemiol 2000; 29: 645-54.

2. Tenderenda M, Jakubik J. Rak przełyku. In: Onkologia. R. Kordek (ed.) Via Medica, Gdańsk 2007; 167-71. [in Polish]

3. Domagała W. Nowotwory. In: Patologia. Stachura J, Domagała W (ed.) Polska Akademia Umiejętności, Kraków 2008; 157-234. [in Polish]

4. Blazeby JM, Avery K, Sprangers M, Pikhart H, Fayers P, Donovan J. Health-related quality of life measurement in randomized clinical trials in surgical oncology. J ClinOncol 2006; 24: 3178-86.

5. Methy N, Bedenne L, Bonnetai, F. Surrogate endpoints for overall survival in digestive oncology trials: which candidates? A questionnaires survey among clinicians and methodologists. BMC Cancer 2010; 10: 277.

6. Velikova G, Booth L, Smith AB, Brown PM, Lynch P, Brown JM, Selby $\mathrm{PJ}$. Measuring quality of life in routine oncology practice improves communication and patient well-being: a randomized controlled trial. J Clin Oncol 2004; 22: 714-24.

7. Aaronson NK, Ahmedzai S, Bergman B, et al. The European Organisation for Research and Treatment of Cancer QLQ-C30: a qualityof-life instrument for use in international clinical trials in oncology. J Natl Cancer Inst 1993; 85: 365-76.

8. Blazeby JM, Conroy T, Hammerlid E, et al. Clinical and psychometric validation of an EORTC questionnaire module, the EORTC QLQ OES18, to assess quality of life in patients with oesophageal cancer. Eur J Cancer 2003; 39: 1384-94.

9. Blazeby JM, Conroy T, Bottomley A, et al. Clinical and psychometric validation of a questionnaire module, the EORTC QLQ-STO 22, to assess quality of life in patients with gastric cancer. Eur J Cancer 2004; 40: $2260-8$

10. Lagergren P, Fayers P, Conroy T, et al. Clinical and psychometric validation of a questionnaire module, the EORTC QLQ-OG25, to assess health-related quality of life in patients with cancer of the oesophagus, the oesophago-gastric junction and the stomach. Eur J Cancer 2007; 43: 2066-73.

11. Conroy T, Marchal F, Blazeby JM. Quality of life in patients with oesophageal and gastric cancer: an overview. Oncology 2006; 70: 391-402.
12. Tomaszewski KA, Püsküllüo lu M, Biesiada K, Bochenek J, Nieckula J, Krzemieniecki K. Validation of the Polish version of the EORTC QLQ-C30 and the QLQ-OG25 for the assessment of health-related quality of life in patients with oesophago-gastric cancer. J Psychosoc Oncol 2013; 31: 191-203.

13. Dewolf L, Koller M, Velikova G, Johnson C, Scott N, Bottomley A; on behalf of the EORTC Quality of Life Group. EORTC Quality Of Life Group Translation Procedure, 3rd edn. European Organisation for Research and Treatment of Cancer, Brussels, Belgium 2009.

14. Fayers P, Aaronson NK, Bjordal K, Curran D, Groenvold M. The EORTC QLQ-C30 Scoring Manual (3rd ed). European Organisation for Research and Treatment of Cancer, Brussels 2001.

15. Blazeby JM. Scoring Procedure for the EORTC QLQOG25. European Organisation for Research and Treatment of Cancer, Brussels 2010.

16. Kassam Z, Mackay H, Buckley CA, Fung S, Pintile M, Kim J, Ringash J. Evaluating the impact on quality of life of chemoradiation in gastric cancer. Curr Oncol 2010; 17: 88-4

17. Munene G, Francis W, Garland SN, Pelletier G, Mack LA, Bathe OF. The quality of life trajectory of resected gastric cancer. J Surg Oncol 2012; 105: 337-41.

18. Reynolds JV, McLaughlin R, Moore J, Rowley S, Ravi N, Byrne PJ. Prospective evaluation of quality of life in patients with localized oesophageal cancer treated by multimodality therapy or surgery alone. Br J Surg 2006; 93: 1084-90.

19. Van der Schaaf M, Derogar M, Lagergren P. Reference values of oesophago-gastric symptoms (EORTC QLQ-OG25) in a populationbased setting. Eur J Cancer 2012; 48: 1602-7.

20. Racine M, Tousignant-Laflamme Y, Kloda LA, Dion D, Dupuis G, Choiniere $M$. A systematic literature review of 10 years of research on sex/gender and experimental pain perception - Part 1: Are there really differences between women and men? Pain 2012; 153: 602-18.

21. Chmielowska K, Tomaszewski KA, Pogrzebielski A, Brandberg Y, Romanowska-Dixon B. Translation and validation of the Polish version of the EORTC QLQ-OPT30 module for the assessment of health-related quality of life in patients with uveal melanoma. Eur J Cancer Care (Engl) 2013; 22: 88-96.

22. Orpana HM, Ross N, Feeny D, McFarland B, Bernier J, Kaplan M. The natural history of health-related quality of life: a 10-year cohort study. Health Rep 2009; 20: 29-35.

23. Ońate-Ocańa LF, Velázquez-Monroy N, Vázquez L, et al. Clinical validation of the EORTC QLQ-OG25 questionnaire for the evaluation of health-related quality of life in Mexican patients with esophagogastric cancers. Psycho-Oncology 2012; 21: 745-53.

24. Allison PJ, Locker D, Wood-Daphinee S. Correlates of health-related quality of life in upper aerodigestive tract cancer patients. Qual Life Res 1998; 7: 713-22.

25. Ockenga J, Valentini L. Review article: anorexia and cachexia in gastrointestinal cancer. Aliment Pharmacol Ther 2005; 22: 583-94.

26. Ravasco P, Monteiro-Grillo I, Vidal PM, Camilo ME. Cancer: disease and nutrition are key determinants of patients' quality of life. Support Care Cancer 2004; 12: 246-52.

27. Krstev S, Dosemeci M, Lissowska J, Chow WH, Zatonski, W, Ward MH. Occupation and risk of stomach cancer in Poland. Ooccup Environ Med 2005; 62: 318-24

28. Didkowska J, Wojciechowska U, Zatoński W. Stomach cancer. In: Prediction of cancer incidence and mortality in Poland up to the year 2025. J. Didkowska, U. Wojciechowska, W. Zatoński (ed.) M. Sklodowska-Curie Institute of Oncology, Warszawa 2009; 19-21.

\section{Address for correspondence}

\section{Krzysztof Tomaszewski MD}

Department of Anatomy,

Jagiellonian University Medical College

Kopernika 12

31-034 Krakow, Poland

tel/fax +48124229511

e-mail: krtomaszewski@gmail.com

Submitted: $\quad 27.12 .2012$

Accepted: $\quad 08.02 .2013$ 